\title{
Self-induced flows enhance the levitation of Leidenfrost drops on liquid baths
}

Sobac, Benjamin; Maquet, Laurent; Duchesne, Alexis; Machrafi, Hatim; Rednikov, Alexey; Dauby, Pierre; Colinet, Pierre; Dorbolo, Stephane

Published in:

Physical Review Fluids

Link to article, DOI:

10.1103/PhysRevFluids.5.062701

Publication date:

2020

Document Version

Publisher's PDF, also known as Version of record

Link back to DTU Orbit

Citation (APA):

Sobac, B., Maquet, L., Duchesne, A., Machrafi, H., Rednikov, A., Dauby, P., Colinet, P., \& Dorbolo, S. (2020). Self-induced flows enhance the levitation of Leidenfrost drops on liquid baths. Physical Review Fluids, 5(6), [062701]. https://doi.org/10.1103/PhysRevFluids.5.062701

\section{General rights}

Copyright and moral rights for the publications made accessible in the public portal are retained by the authors and/or other copyright owners and it is a condition of accessing publications that users recognise and abide by the legal requirements associated with these rights.

- Users may download and print one copy of any publication from the public portal for the purpose of private study or research.

- You may not further distribute the material or use it for any profit-making activity or commercial gain

- You may freely distribute the URL identifying the publication in the public portal 


\title{
Self-induced flows enhance the levitation of Leidenfrost drops on liquid baths
}

\author{
Benjamin Sobac $\odot,{ }^{1,}$ Laurent Maquet, ${ }^{2}$ Alexis Duchesne $\odot,{ }^{2,3}$ Hatim Machrafi $\odot,{ }^{4}$ \\ Alexey Rednikov $\odot,{ }^{1}$ Pierre Dauby $\odot,{ }^{4}$ Pierre Colinet, ${ }^{1}$ and Stéphane Dorbolo $\circledast^{2}$ \\ ${ }^{1}$ TIPs Laboratory, Université libre de Bruxelles, Brussels, Belgium \\ ${ }^{2}$ GRASP, Université de Liège, Liège, Belgium \\ ${ }^{3}$ Department of Physics, Technical University of Denmark, Lyngby, Denmark \\ ${ }^{4}$ TPI, Université de Liège, Liège, Belgium
}

(Received 29 June 2019; accepted 28 April 2020; published 9 June 2020)

\begin{abstract}
The Leidenfrost effect, classically associated with drops levitating on their own vapor over hot solid surfaces, can also be observed over hot baths of nonvolatile liquids. In view of substrate fluidity, heat transfer through the bath to the drop should most certainly be dominated by convection and not by only conduction as in the solids, which may be instrumental for an efficient heat supply to the drop given typically poor thermal conductivity of the liquids. Here, we undertake an experimental and numerical study of the flow in a bath of silicone oil V20 induced by an overlying Leidenfrost drop, highlighting that a toroidal vortex is formed underneath the drop whose direction of circulation turns out to be different for drops of different liquids. We show that this is due to a shift in a delicate interplay between three mechanisms pulling in different directions: (i) shear stresses exerted by the vapor escaping from the gap between the bath and the drop, as well as (ii) buoyancy action and (iii) thermocapillary (Marangoni) stresses, both due to local evaporative cooling of the bath by the drop. Whatever the structure of this locally induced convection, its crucial heat transfer enhancing efficiency is readily confirmed in numerical simulations as favoring levitation.
\end{abstract}

DOI: 10.1103/PhysRevFluids.5.062701

When a liquid drop is deposited over a solid surface whose temperature is sufficiently above the boiling point of the liquid (the difference referred to as the superheat), the drop does not experience nucleate boiling but rather levitates over a thin layer of its own vapor [1,2]. This is known as the Leidenfrost effect. While highly undesirable in certain cooling or boiling applications [3,4], because of a drastic decrease of the energy transferred between the solid and the evaporating liquid due to poor thermal conductivity of the vapor, this effect can be of great interest in many other processes profiting from this absence of contact with the surface that considerably reduces the friction and confers an extreme mobility on the drop. The Leidenfrost effect thus appears to offer significant new opportunities and capabilities, for instance, in self-propelling drops in preferred directions [5-8], in drag reduction using the vapor layer [9,10], in the frictionless transport of small compounds [11], in chemical reactors without borders [12,13], and in heat engines [14].

While the classical Leidenfrost effect, on solids, has thus far attracted the most attention (see the review in Ref. [2], or Refs. [15-17] for recent discoveries), it is less known that the Leidenfrost state can also manifest over (nonvolatile) liquid baths [18-20], as illustrated in Fig. 1(a). However, a liquid substrate offers several advantages compared to a solid one for the Leidenfrost phenomenon and related applications as explained below. Astonishingly, while the Leidenfrost state can only be

*bsobac@ulb.ac.be 

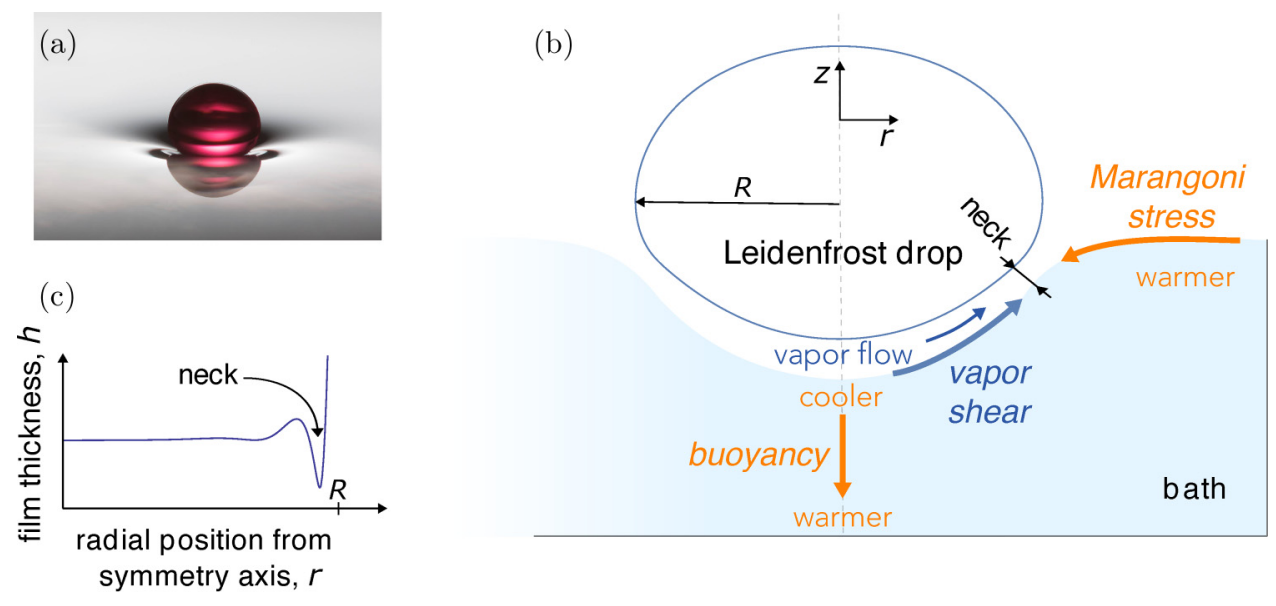

(d)

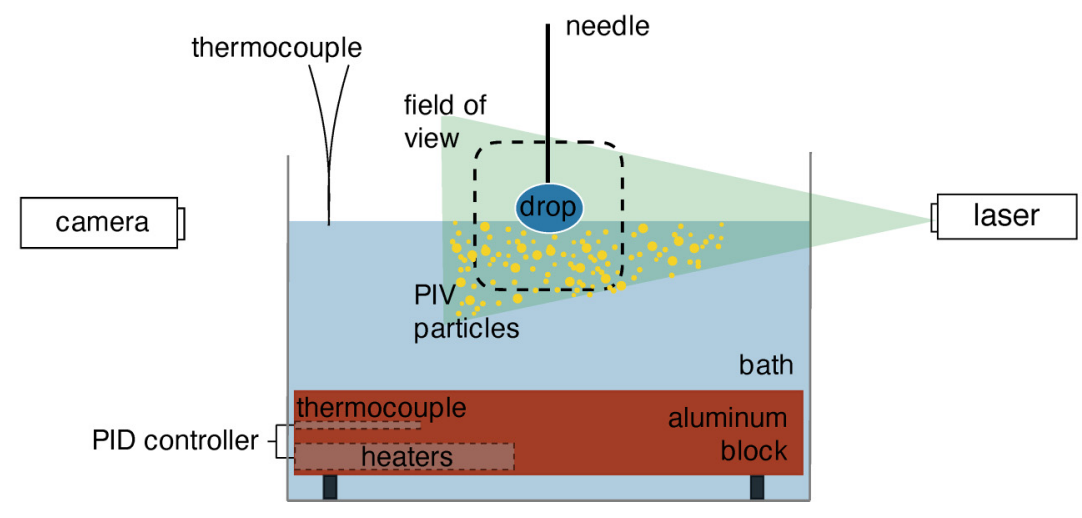

FIG. 1. (a) Leidenfrost drop of (dyed) ethanol of $R=1.2 \mathrm{~mm}$ on a silicone oil V20 bath at $T_{\text {bath }}=79^{\circ} \mathrm{C}$ $\left(\Delta T=1{ }^{\circ} \mathrm{C}\right)$. (b) System sketch with various bath-flow mechanisms and (c) the typical vapor gap profile [20,23]. (d) Experimental setup.

realized at important superheats (of the order of tens of degrees or more) on solids, it can be obtained at much smaller ones on liquid baths. For instance, while $80^{\circ} \mathrm{C}$ of superheat is necessary for an ethanol drop to levitate on an aluminum substrate [21], $1^{\circ} \mathrm{C}$ of superheat turns out to be already sufficient for its levitation on a 20-cSt silicone oil bath [20]. One reason for such a drastic decrease of critical superheat is likely due to the surface roughness of the substrate (it is generally reported that the higher the roughness, the higher the critical superheat [4,22]), which is molecularly smooth in the case of a liquid bath. This condition is probably necessary but not sufficient in light of recent experiments performed on wafers that are extremely smooth, too [17]. Another consequence of a liquid substrate is its surface deformability under the drop's weight. This results in a very different morphology of the vapor gap (recently investigated in detail in Ref. [23]) as compared to the case of a flat solid substrate (cf. Ref. [24]): The gap appears to be nearly uniform and exhibits undulations only towards a thin neck, as sketched in Fig. 1(c). Furthermore, substrate deformation leads to stabilization by means of suppressing the chimney instability [23], hence enabling the manipulation of larger drops. Similar configurations, involving a drop levitating over a liquid substrate, have also been reported in somewhat different situations, when it is the bath but not the levitating object that evaporates (referred to as "inverse Leidenfrost") [25-29], or when the drop and the bath are made of the same liquid (Hickman "boules") [30,31], also manifesting interesting specificities related to the use of a liquid bath. 
TABLE I. Molar mass $\hat{M}$; liquid density $\rho$, latent heat $\mathcal{L}$, surface tension $\gamma$, and capillary length $\ell_{c}=$ $\sqrt{\gamma / \rho g}$ ( $g$ gravity acceleration) at $T_{\text {sat }}$ (at $\sim 90^{\circ} \mathrm{C}$ for oil [34]); vapor thermal conductivity $\lambda_{v}$ and viscosity $\mu_{v}$ at $\left(T_{\text {sat }}+\frac{1}{2} \Delta T\right)$ and $1 \mathrm{~atm}$, where $\Delta T=19^{\circ} \mathrm{C}$.

\begin{tabular}{lrrrrrrrr}
\hline \hline Liquids & $\hat{M}(\mathrm{~g} / \mathrm{mol})$ & $T_{\text {sat }}\left({ }^{\circ} \mathrm{C}\right)$ & $\rho\left(\mathrm{kg} / \mathrm{m}^{3}\right)$ & $\mathcal{L}(\mathrm{kJ} / \mathrm{kg})$ & $\gamma(\mathrm{mN} / \mathrm{m})$ & $\ell_{c}(\mathrm{~mm})$ & $\lambda_{v}[\mathrm{~mW} /(\mathrm{m} \mathrm{K})]$ & $\mu_{v}(\mu \mathrm{Pas})$ \\
\hline Ethanol & 46 & 79 & 728 & 840 & 17 & 1.56 & 17.3 & 10.7 \\
HFE-7100 & 250 & 61 & 1435 & 112 & 10 & 0.85 & 10.9 & 12.7 \\
Silicone oil & $\sim 3000$ & & 896 & & 20 & 1.51 & & \\
\hline \hline
\end{tabular}

In this Rapid Communication, we are concerned with another consequence of substrate fluidity ignored so far: the flows inside the underlying liquid substrate. We report about flows caused in the bath by the levitating Leidenfrost drop and reveal various hydrodynamic structures originating from a delicate interplay between three mechanisms. Remarkably, whatever the structure of these flows, they result in a positive feedback favoring the levitation of the drop.

We implemented particle image velocimetry (PIV) to measure the flow in the liquid bath below a Leidenfrost drop [Fig. 1(d)]. A 8-cm cubic glass aquarium with a heating aluminum plate $\left(8 \times 8 \times 2 \mathrm{~cm}^{3}\right)$ at its bottom was filled with silicone oil V20 (20 cSt at normal conditions) up to $4 \mathrm{~cm}$ above the heater and seeded with fluorescent particles $\left(15 \mu \mathrm{m}\right.$ diameter, $1100 \mathrm{~kg} / \mathrm{m}^{3}$ density) made of a polymer shell surrounding a rhodamine core. A continuous laser (Dantec RayPower 2000, $532 \mathrm{~nm}$ ) was used to create a sheetlike beam passing through the drop's axis, and an orthogonally placed high-speed camera (Phantom v9.1) was used to record particles. A PID controller and a thermocouple at the bottom permitted us to control the temperature at the heater. Another thermocouple was placed just below the surface of the oil layer. To ensure characterizing bath flows only resulting from the presence of a Leidenfrost drop, a bath as isothermal as possible is desired prior to drop deposition. To this purpose, the bath was initially heated above the desired temperature $T_{\text {bath }}\left(>T_{\text {sat }}\right.$ the boiling temperature of the drop). Then, with the heater off, it was allowed to cool down to $\sim T_{\text {bath }}$, the measured temperature difference between the bottom and the top not exceeding $1-3{ }^{\circ} \mathrm{C}$ and the measured maximum velocity of natural convection in the bath not exceeding one tenth of the maximal velocity measured later on in the presence of a drop. A Leidenfrost drop was then generated above the bath at the tip of a needle continuously fed by a syringe pump, enabling both to immobilize the drop and to study a quasisteady situation with a drop of constant size, similarly to what accomplished over a solid substrate in Refs. [32,33].

We considered drops of two different liquids, ethanol and HFE-7100, whose properties are reported in Table I. Surprisingly, we observe two markedly different behaviors for these two liquids. Figures 2(a1), 2(a2), 2(b1), and 2(b2) show the experimental streamlines and reconstructed velocity fields within the bath underlying Leidenfrost drops of dimensionless radius $R / \ell_{c} \approx 2$ subjected to a superheat $\Delta T=19^{\circ} \mathrm{C}$. In both cases, the experiments reveal quasiaxisymmetric flow patterns, which must be due to the presence of the drop. Disregarding the small nonaxisymmetric disturbances, one can discern in both cases a circulation in the form of a toroidal vortex. However, the direction of the circulation turns out to be just the opposite: an inward radial flow along the bath surface and a sinking plume in the ethanol case [Fig. 2(a2)], and a completely reversed flow, upwards at the symmetry axis and outwards at the surface, in the HFE-7100 case [Fig. 2(b2)]. Maximum velocities ( $\sim$ several $\mathrm{cm} / \mathrm{s}$, slightly higher in the HFE-7100 case) are attained inside the plume in the bath bulk in the ethanol case, but near the surface not far from the drop in the HFE-7100 case.

What are the actual mechanisms controlling the flow in the bath [see Fig. 1(b)] and explaining its possible reversal? First, the vapor generated by drop evaporation is evacuated from the gap between the drop and the substrate by means of a radial outward vapor flow [20,23]. Vapor shear stress thereby exerted on the bath surface below the drop favors the direction of circulation observed in the case of an HFE-7100 drop. Second, a Leidenfrost drop requires energy for its evaporation, which 


\section{Experimental results}
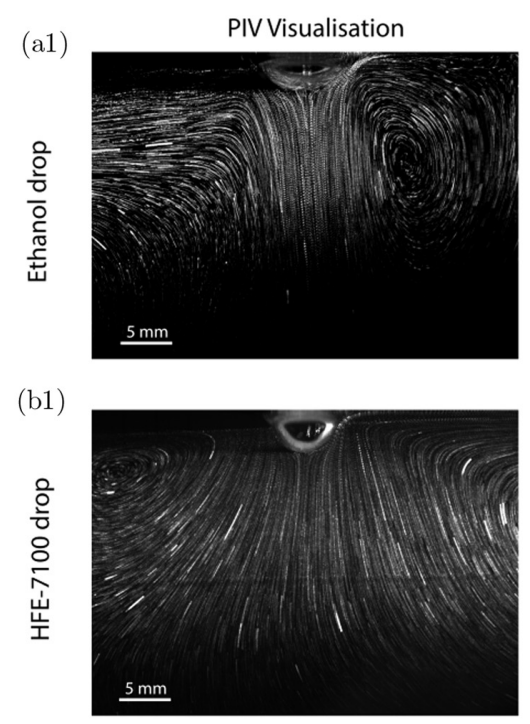

(a2)

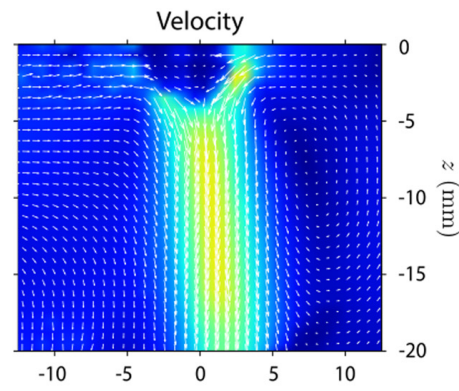

(b2)
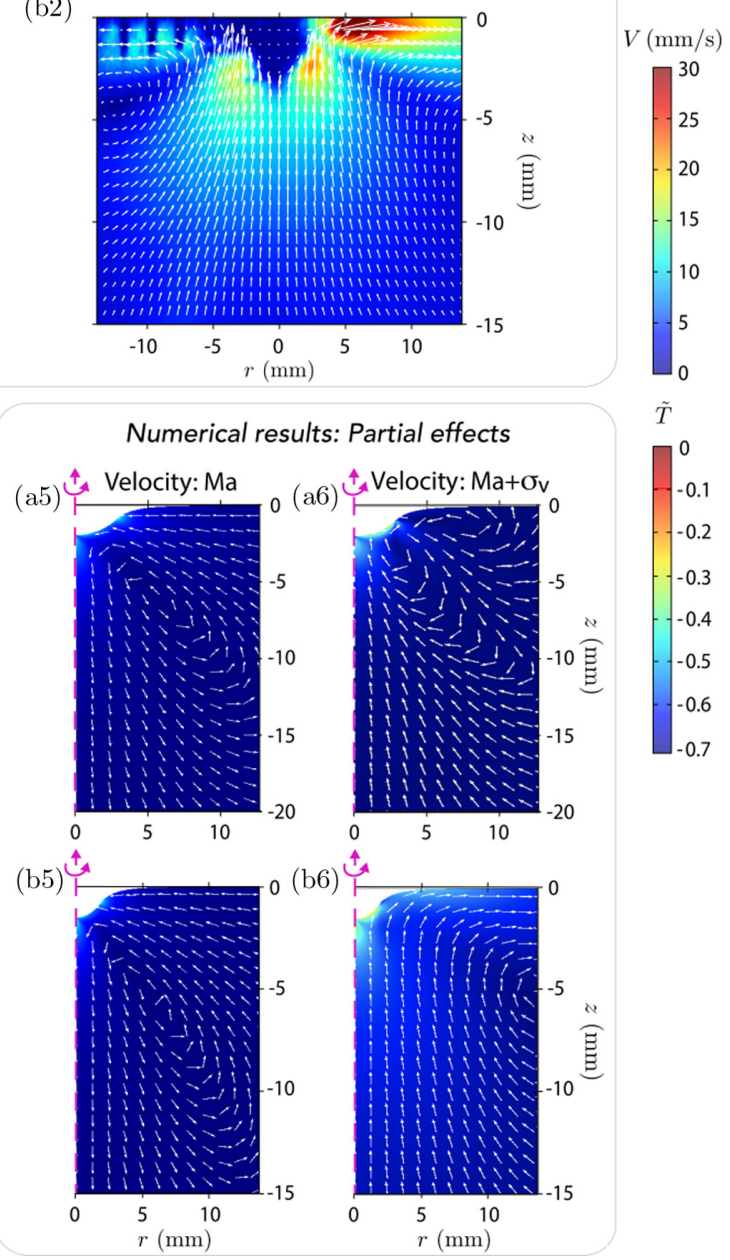

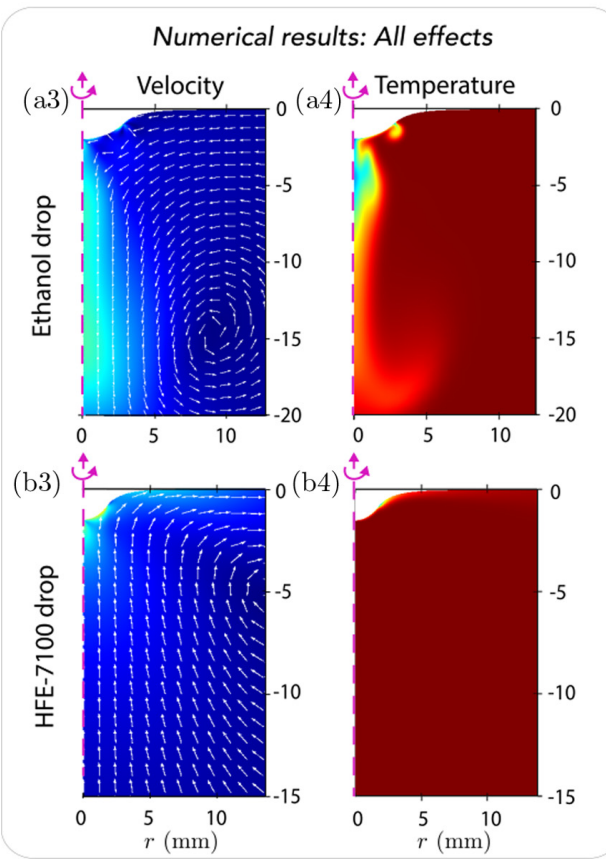

FIG. 2. Flow and temperature fields in the bath in the cases of ethanol (a) and HFE-7100 (b) Leidenfrost drops, both of radius $R=2 \ell_{\mathrm{c}}$ and subjected to a superheat $\Delta T=19^{\circ} \mathrm{C}$ over the corresponding saturation temperature (given in Table I). PIV images (a1 and b1), postprocessed experimental images (a2 and b2), numerical results in the same format with all effects included (a3, a4, b3, and b4), with only the thermal Marangoni effect included but the vapor shear stress and buoyancy excluded (a5 and b5), with the thermal Marangoni effect and vapor shear stress included but buoyancy excluded (a6 and b6). The dimensionless temperature is defined as $\tilde{T}=\left(T-T_{\text {bath }}\right) /\left(T_{\text {bath }}-T_{\text {sat }}\right)$. Note that the laser sheet that illuminates the PIV particles comes from the right in (a1) and (b1), and is obstructed by the surface deformation under the drop. 

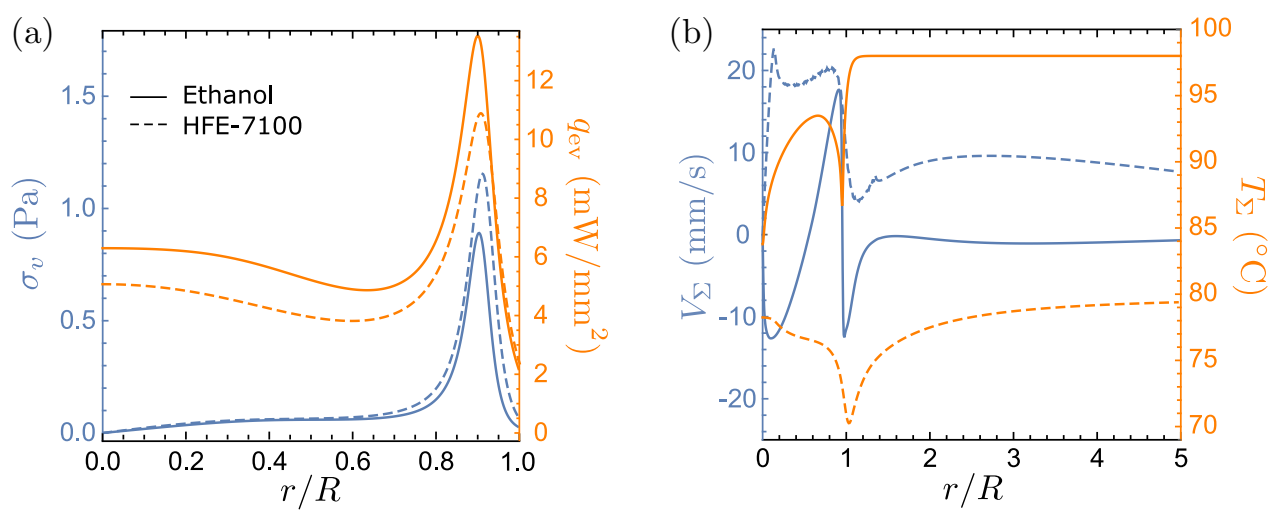

FIG. 3. Profiles of (a) the vapor shear stress and evaporation heat flux, obtained using the model by Maquet et al. [20], and (b) the surface tangential velocity and temperature, computed here with all effects included, for the two cases of Fig. 2. The stress and velocity are positive when directed outwards.

is mainly transferred by conduction across the vapor gap from the substrate, hence cooling down the underlying liquid bath. Such a local cooling, exacerbated by poor thermal conductivity of the substrate [33], could be responsible for the direction of circulation observed in the case of an ethanol drop both by potentially giving rise to a sinking buoyant plume of cooler (hence heavier) liquid beneath the drop, and by means of a surface-tension gradient (Marangoni stresses) directed inwards along the bath surface (towards a cooler spot under the drop, where the surface tension, a decreasing function of temperature, is higher). Although the direction of the Marangoni stresses immediately underneath the drop is a priori less obvious (due to a stronger cooling expected at the neck, the location of the most intense evaporation [33]), we shall confirm later on that the aforementioned inward tendency is overall predominant for the Marangoni contribution.

To rationalize the experimental results and to better understand the interplay between these various mechanisms, corresponding axisymmetric numerical simulations were performed. On the one hand, for each given $R, \Delta T=T_{\text {bath }}-T_{\text {sat }}$, and liquid combination, the model introduced and successfully validated with experiments in Ref. [20] and further improved in Ref. [23] was used to compute (i) the shape of the bath surface underneath the drop, (ii) the distribution of the vapor shear stress $\sigma_{v}$ exerted upon the bath surface in the gap, and (iii) the distribution of the heat flux $q_{\mathrm{ev}}$ across the gap (see Ref. [35] for more details of the model [20,23]).

The results for $\sigma_{v}$ and $q_{\mathrm{ev}}$ for the two baseline cases considered here are shown in Fig. 3(a). Both $\sigma_{v}$ and $q_{\mathrm{ev}}$ are maximum at the neck and quickly decay towards the periphery of the area covered by the drop. In our modeling, they are set equal to zero for $r / R>1$. On the other hand, for the flow and temperature fields in the bath, the full Navier-Stokes equations in the Boussinesq approximation coupled with the convective heat equation are solved [34]. For simplicity, the bath was assumed cylindrical (diameter $8 \mathrm{~cm}$ and depth $4 \mathrm{~cm}$ ). At the free surface [of a predetermined shape; see item (i) above], impermeability, a tangential stress [a sum of $\sigma_{v}$ determined in item (ii) above and the thermal Marangoni stress], and a heat loss $\left(-q_{\mathrm{ev}}\right)$ determined in item (iii) above are imposed. Most parts of the bath surface, away from the drop, are hereby practically horizontal, stress free, and insulating. Impermeability, no slip and insulation are imposed at the other boundaries, except for the bottom kept at a fixed temperature $T_{\text {bath }}$. COMSOL MULTIPHYSICS was used (unsteady solver until attaining a steady state).

The simulations, with the two baseline results shown in Figs. 2(a3), 2(b3), and 3(b), are seen to capture well both the flow patterns and the orders of magnitude of the velocities observed in experiments. Quite remarkably, this includes the direction of circulation and its reversal between the ethanol and HFE-7100 cases. This indicates (in the framework of our earlier reasoning) that it is the vapor shear that predominates in the latter and the cooling-induced thermal convective factors 
in the former case. The simulated temperature fields [Figs. 2(a4), 2(b4), and 3(b)] turn out to be drastically different and reveal a strong contrast underlying the opposite direction of circulation in the two cases. In the case of an HFE-7100 drop, the bulk of the bath is quasi-isothermal: The predominant vapor shear wipes the cooler liquid from all over the bath surface underneath the drop within a boundary-layer-like structure [Fig. 2(b4)], thereby preventing the formation of a plume. In contrast, in the case of an ethanol drop, the cooler liquid penetrates deep into the bulk of the bath by means of a thermal plume [Fig. 2(a4)]. In both cases, however, an important cooling occurs near the neck location [Figs. 2(a4), 2(b4), and Fig. 3(b)].

The reason why it is the vapor shear that predominates in the HFE-7100 case and the thermal convective factors in the ethanol case can be grasped from Fig. 3(a). Indeed, one can readily see that the heat flux $q_{\mathrm{ev}}$ turns out to be higher whereas the shear stress $\sigma_{v}$ is lower in the latter case than in the former. This goes perfectly along with our findings for the direction of circulation. Test simulations in which $q_{\mathrm{ev}}$ was artificially decreased by a factor of $3 / 2$ for ethanol and increased by the same factor for HFE-7100 both manifested a circulation reversal with respect to the original settings, further consolidating our conclusions.

When the thermal convective factors, namely buoyancy and the Marangoni effect, prevail in setting the direction of circulation, one can wonder which is the role of each of them separately. Test simulations were performed in which we could "switch off" any one or two of the three mechanisms involved (vapor shear, Marangoni, and buoyancy). First of all, given the aforementioned uncertainty about the direction of the Marangoni stresses immediately underneath the drop, a computation retaining exclusively the Marangoni mechanism confirmed the earlier reasoning as far as the overall inward tendency is concerned, as shown in Figs. 2(a5) and 2(b5). Next, even in the ethanol case (not to mention the HFE-7100 one), it turned out that the buoyancy alone (the Marangoni mechanism being switched off) has no chance against the vapor shear: The latter predominates by wiping off the cooler liquid before a sinking plume could be formed. In contrast, in the ethanol case, the Marangoni effect alone (buoyancy being switched off) partly succeeds against the vapor shear, reversing the flow over the bath surface not covered by the drop, as shown in Fig. 2(a6). The corresponding flow field in the HFE-7100 case, when the Marangoni effect is not capable of doing so, is displayed for comparison in Fig. 2(b6). However, the flow pattern of Fig. 2(a6) is still far from the one shown in Fig. 2(a3), and here comes the role of buoyancy: Once the Marangoni effect has fulfilled its role at the surface as in Fig. 2(a6), the buoyancy adds a final touch by gathering the cooler liquid, leaving the surface boundary layer, into a falling plume as in Fig. 2(a3). This elucidates the specific contribution of each of the three mechanisms in the observed flow pattern.

Note however that, in the ethanol case, even if the vapor shear is not strong enough to determine the direction of circulation globally, it still seems to prevail locally in a tiny cell (toroidal vortex) formed at the surface underneath the drop right before the neck, where the direction of circulation is opposite to the global one [cf. Fig. 2(a3) and the sign change of $V_{\Sigma}$ in Fig. 3(b)]. This is a simulation result, and it remains unclear whether such a tiny cell also exists in experiments while being merely indiscernible.

Finally, what is the back influence of the bath flow and temperature fields induced by the drop on the Leidenfrost effect itself? Since the bath flow velocities $(\sim \mathrm{cm} / \mathrm{s})$ are much smaller than those of the vapor in the gap (up to $\sim 1 \mathrm{~m} / \mathrm{s}$ ), a mechanical influence appears to be negligible (and no effects of the kind [36,37] are essential here). However, the influence turns out to be important thermally. The existence of a critical Leidenfrost superheat implies that a Leidenfrost drop can only be realized under the conditions of a sufficient energy supply, hence the importance of convective heat transport in the bath due to the flow. Indeed, a hemispherical-sink-based estimation $2 \pi \lambda_{\text {eff }} \Delta T_{\text {bath }} R \sim Q_{\mathrm{ev}}$ with $Q_{\mathrm{ev}}=\iint q_{\mathrm{ev}} d S$ (integration over the bath surface) and $\Delta T_{\text {bath }} \sim 0.5 \Delta T$ the typical temperature difference in the bath obtained in our computation [cf. Figs. 2(a4) and 2(b4)] yields an effective thermal conductivity of the medium $\lambda_{\text {eff }} \sim 2 \mathrm{~W} /(\mathrm{m} \mathrm{K})$ in both the ethanol and HFE-7100 cases (irrespective of the circulation direction). This represents a $\sim 14$-fold enhancement over the (poor) thermal conductivity of silicone oil $[\lambda \sim 0.14 \mathrm{~W} /(\mathrm{m} \mathrm{K})]$. Estimation of the thermal Péclet number $\mathrm{Pe}=v R / \kappa \sim 400$ [with $\kappa=0.1 \mathrm{~mm}^{2} / \mathrm{s}$ the thermal diffusivity of 
silicone oil, $v \sim 1.5 \mathrm{~cm} / \mathrm{s}$ and $R \sim 0.3 \mathrm{~cm}$ for the ethanol case, and $v \sim 2 \mathrm{~cm} / \mathrm{s}$ and $R \sim 0.2 \mathrm{~cm}$ for the HFE-7100 case; cf. Figs. 2(a3) and 2(b3)] points to the same order of magnitude of convective enhancement $\left(\sim \mathrm{Pe}^{1 / 2} \sim 20\right.$ in accordance with the thermal boundary layer considerations). If the flow were absent and heat transfer were purely conductive, the Leidenfrost state apparently would no longer be possible (due to an insufficient heat flux). This is probably why the attempts to generate a Leidenfrost drop at $\Delta T \sim 1 \mathrm{~K}$ for a series of bath liquids of an increasing viscosity ceased to be successful above a certain viscosity [20], when an effective convective transport was already supposedly suppressed by large viscosity.

Thus far, we focused on the experimental reference cases $\left(R \approx 2 \ell_{c}\right.$ and $\left.\Delta T \approx 19^{\circ} \mathrm{C}\right)$, which permitted us to grasp the essence of the phenomenon. To complement the picture, an additional parametric study has been undertaken by means of our numerical procedure in the neighboring drop-size and superheat ranges $\left(1 \lesssim R / \ell_{c} \lesssim 4\right.$ and $\left.5{ }^{\circ} \mathrm{C} \lesssim \Delta T \lesssim 50^{\circ} \mathrm{C}\right)$ for both HFE-7100 and ethanol Leidenfrost drops. For HFE-7100, the induced flow pattern in the bath does not essentially change in this range of parameters as compared to the reference case. For ethanol, in contrast, the flow structure turns out to be less robust and does change, the change being essentially related to the earlier mentioned tiny cell (toroidal vortex): This cell seems to fully disappear for smaller drops, but can appreciably grow in one direction or another for larger drops and/or larger or smaller superheat (see Supplemental Material for these and other details [38]). Regardless of the flow structure, the convective enhancement of heat transfer through the bath always remains there and is important. It reaches the values of $\sim 40$ for larger $R$ and $\Delta T$, but falls down to 2 for smaller $R$ and $\Delta T$. The question of the enhancement reduction at such smaller superheat is mingled with the not yet understood issue of the critical Leidenfrost threshold, and its interpretation is therefore left beyond the scope of the present Rapid Communication. On the other hand, let us note that the enhancement reduction for smaller drops seems to go along with the observation [20] that, for a given $\Delta T$, sufficiently small drops could not remain in levitation and fell down on the bath (which is not what is observed on solid substrates).

To conclude, we have evidenced a peculiar existential feedback: The Leidenfrost drop induces a flow in the bath, which in turn ensures a sufficient energy supply to the drop so as to sustain the Leidenfrost state. The structure of the bath flow, and in particular the direction of circulation, is herewith not universal and depends on the interplay between the opposing actions of the vapor shear stress $\sigma_{v}$ and the evaporative cooling $q_{\mathrm{ev}}$ (giving rise to the thermal Marangoni and buoyancy contributions). The outcome depends on the material of the liquids involved (e.g., HFE-7100 or ethanol here), as well as the superheat $\Delta T$ and the drop size $R$ since the ratio $\sigma_{v} / q_{\mathrm{ev}}$ varies with both $\Delta T$ and $R$ [20,35]. All these observations, rationalized owing to numerical simulations, may be of importance for understanding the Leidenfrost threshold or the (collective) motion of such drops at the surface of the bath $[19,28,29,39]$.

The authors gratefully acknowledge the support of Fonds de la Recherche Scientifique-FNRS (Postdoctoral Research Position of B.S., Senior Research Associate Position of S.D., and Research Director Position of P.C.), ESA-BELSPO (MAP and PRODEX projects), BELSPO (IAP 7/38 $\mu$ MAST project), and People Programme (Marie Curie Actions) of the European Union's Seventh Framework Programme (FP7/2007-2013) under REA Grant Agreement No. 609405 (COFUNDPostdocDTU).

[1] J. G. Leidenfrost, De Aquae Communis Nonnullis Qualitatibus Tractatus (Ovenius, Duisburg, 1756).

[2] D. Quéré, Leidenfrost dynamics, Annu. Rev. Fluid Mech. 45, 197 (2013).

[3] V. K. Dhir, Boiling heat transfer, Annu. Rev. Fluid Mech. 30, 365 (1998).

[4] H. Kim, B. Truong, J. Buongiorno, and L.-W. Hu, On the effect of surface roughness height, wettability, and nanoporosity on Leidenfrost phenomena, Appl. Phys. Lett. 98, 083121 (2011). 
[5] H. Linke, B. J. Alemán, L. D. Melling, M. J. Taormina, M. J. Francis, C. C. Dow-Hygelund, V. Narayanan, R. P. Taylor, and A. Stout, Self-Propelled Leidenfrost Droplets, Phys. Rev. Lett. 96, 154502 (2006).

[6] G. Lagubeau, M. Le Merrer, C. Clanet, and D. Quéré, Leidenfrost on a ratchet, Nat. Phys. 7, 395 (2011).

[7] Á. G. Marín, D. Arnaldo del Cerro, G. R. B. E. Römer, B. Pathiraj, A. Huis in 't Veld, and D. Lohse, Capillary droplets on Leidenfrost micro-ratchets, Phys. Fluids 24, 122001 (2012).

[8] B. Sobac, A. Rednikov, S. Dorbolo, and P. Colinet, Self-propelled Leidenfrost drops on a thermal gradient: A theoretical study, Phys. Fluids 29, 082101 (2017).

[9] I. U. Vakarelski, J. O. Marston, D. Y. C. Chan, and S. T. Thoroddsen, Drag Reduction by Leidenfrost Vapor Layers, Phys. Rev. Lett. 106, 214501 (2011).

[10] I. U. Vakarelski, N. A. Patankar, J. O. Marston, D. Y. C. Chan, and S. T. Thoroddsen, Stabilization of Leidenfrost vapour layer by textured superhydrophobic surfaces, Nature (London) 489, 274 (2012).

[11] A. Hashmi, Y. Xu, B. Coder, P. A. Osborne, J. Spafford, G. E. Michael, G. Yu, and J. Xu, Leidenfrost levitation: Beyond droplets, Sci. Rep. 2, 797 (2012).

[12] M. Elbahri, D. Paretkar, K. Hirmas, S. Jebril, and R. Adelung, Anti-lotus effect for nanostructuring at the Leidenfrost temperature, Adv. Mater. 19, 1262 (2007).

[13] R. Abdelaziz, D. Disci-Zayed, M. K. Hedayati, J.-H. Pöhls, A. U. Zillohu, B. Erkartal, V. S. K. Chakravadhanula, V. Duppel, L. Kienle, and M. Elbahri, Green chemistry and nanofabrication in a levitated Leidenfrost drop, Nat. Commun. 4, 2400 (2013).

[14] G. G. Wells, R. Ledesma-Aguilar, G. McHale, and K. Sefiane, A sublimation heat engine, Nat. Commun. 6, 6390 (2015).

[15] A. Bouillant, T. Mouterde, P. Bourrianne, A. Lagarde, C. Clanet, and D. Quéré, Leidenfrost wheels, Nat. Phys. 14, 1188 (2018).

[16] S. Lyu, V. Mathai, Y. Wang, B. Sobac, P. Colinet, D. Lohse, and C. Sun, Final fate of a Leidenfrost droplet: Explosion or takeoff, Sci. Adv. 5, eaav8081 (2019).

[17] P. Bourrianne, C. Lv, and D. Quéré, The cold Leidenfrost regime, Sci. Adv. 5, eaaw0304 (2019).

[18] A. Snezhko, E. B. Jacob, and I. S Aranson, Pulsating-gliding transition in the dynamics of levitating liquid nitrogen droplets, New J. Phys. 10, 043034 (2008).

[19] M. Le Merrer, C. Clanet, D. Quéré, É. Raphäl, and F. Chevy, Wave drag on floating bodies, Proc. Natl. Acad. Sci. USA 108, 15064 (2011).

[20] L. Maquet, B. Sobac, B. Darbois-Texier, A. Duchesne, M. Brandenbourger, A. Rednikov, P. Colinet, and S. Dorbolo, Leidenfrost drops on a heated liquid pool, Phys. Rev. Fluids 1, 053902 (2016).

[21] A.-B. Wang, C.-H. Lin, and C.-C. Chen, The critical temperature of dry impact for tiny droplet impinging on a heated surface, Phys. Fluids 12, 1622 (2000).

[22] J. D. Bernardin and I. Mudawar, The Leidenfrost point: Experimental study and assessment of existing models, J. Heat Transfer 121, 894 (1999).

[23] M. A. J. van Limbeek, B. Sobac, A. Rednikov, P. Colinet, and J. H. Snoeijer, Asymptotic theory for a Leidenfrost drop on a liquid pool, J. Fluid Mech. 863, 1157 (2019).

[24] B. Sobac, A. Rednikov, S. Dorbolo, and P. Colinet, Leidenfrost effect: Accurate drop shape modeling and refined scaling laws, Phys. Rev. E 90, 053011 (2014).

[25] H. Kim, Floating phenomenon of a water drop on the surface of liquid nitrogen, J. Korean Phys. Soc. 49, L1335 (2006).

[26] Y. S. Song, D. Adler, F. Xu, E. Kayaalp, A. Nureddin, R. M. Anchan, R. L. Maas, and U. Demirci, Vitrification and levitation of a liquid droplet on liquid nitrogen, Proc. Natl. Acad. Sci. USA 107, 4596 (2010).

[27] M. Adda-Bedia, S. Kumar, F. Lechenault, S. Moulinet, M. Schillaci, and D. Vella, Inverse Leidenfrost effect: Levitating drops on liquid nitrogen, Langmuir 32, 4179 (2016).

[28] A. Gauthier, C. Diddens, R. Proville, D. Lohse, and D. van der Meer, Self-propulsion of inverse Leidenfrost drops on a cryogenic bath, Proc. Natl. Acad. Sci. USA 116, 1174 (2019).

[29] A. Gauthier, D. van der Meer, J. H. Snoeijer, and G. Lajoinie, Capillary orbits, Nat. Commun. 10, 3947 (2019).

[30] K. C. D. Hickman, Floating drops and boules, Nature (London) 201, 985 (1964). 
[31] K. C. D. Hickman, J. Maa, A. Davidhazy, and O. Mady, Features floating drops and liquid boules-a further look, Ind. Eng. Chem. 59, 18 (1967).

[32] A.-L. Biance, C. Clanet, and D. Quéré, Leidenfrost drops, Phys. Fluids 15, 1632 (2003).

[33] M. A. J. van Limbeek, M. H. Klein Schaarsberg, B. Sobac, A. Rednikov, C. Sun, P. Colinet, and D. Lohse, Leidenfrost drops cooling surfaces: Theory and interferometric measurement, J. Fluid Mech. 827, 614 (2017).

[34] Silicone oil V20 properties: $\lambda=0.14 \mathrm{~W} /(\mathrm{m} \mathrm{K})$ is the thermal conductivity, $\kappa=0.1 \mathrm{~mm}^{2} / \mathrm{s}$ is the thermal diffusivity, and $\rho=896 \mathrm{~kg} / \mathrm{m}^{3}$ is the density at $\sim 90^{\circ} \mathrm{C}, v=6.57(8.28) \mathrm{cSt}$ is the viscosity at $T_{\text {bath }}=$ $98(80)^{\circ} \mathrm{C}, 0.001 \mathrm{~K}^{-1}$ is the thermal expansion coefficient, and $d \gamma / d T=-0.05 \mathrm{mN} /(\mathrm{mK})$.

[35] The model [20,23] proceeds from the drop and bath shapes as for an equilibrium superhydrophobic drop floating on the bath, except for the gap between them becoming a nonvanishing one filled with vapor, although still very thin (thickness $h$ ). Conduction-limited heat transfer across the gap $q_{\mathrm{ev}}=$ $\lambda_{v} \Delta T / h$ drives vapor generation therein with a density $j_{\mathrm{ev}}=q_{\mathrm{ev}} / \mathcal{L}\left(\mathrm{kg} / \mathrm{m}^{2} \mathrm{~s}\right)$. This vapor is evacuated from the gap by means of a lubrication flow $u=\left(2 \mu_{v}\right)^{-1}\left(\partial_{s} P_{v}\right)\left(y^{2}-h y\right)$ with a volume flux $Q=$ $\int_{0}^{h} u d y=-\left(12 \mu_{v}\right)^{-1} h^{3} \partial_{s} P_{v}$, exerting a stress $\sigma_{v}=\left.\mu_{v} \partial_{y} u\right|_{y=0}=-(1 / 2) h \partial_{s} P_{v}$. Here, $s, y, u$, and $P_{v}$ are the tangential and normal coordinates, tangential velocity, and vapor pressure in the gap. $P_{v}$ is a functional of the geometric characteristics including $h$. It consists only of the hydrostatic and Laplace-pressure contributions coming from the liquid. At the exit from the gap, $P_{v}$ tends to the ambient pressure. The final vapor lubrication equation (quasisteady mass balance) is of the form $r^{-1} \partial_{s}(r Q)=j_{\mathrm{ev}} / \rho_{v}$, whose solution yields $h(s)$ and hence all characteristics of the vapor gap. As a first approximation, the model neglects the (back) influence of the drop-induced flow and temperature nonuniformity in the bath on the Leidenfrost drop and the characteristics (i)-(iii).

[36] P. Dell'Aversana, J. R. Banavar, and J. Koplik, Suppression of coalescence by shear and temperature gradients, Phys. Fluids 8, 15 (1996).

[37] P. Dell'Aversana and G. P. Neitzel, When liquids stay dry, Phys. Today 51(1), 38 (1998).

[38] See Supplemental Material at http://link.aps.org/supplemental/10.1103/PhysRevFluids.5.062701 for the additional parametric study.

[39] S. D. Janssens, S. Koizumi, and E. Fried, Behavior of self-propelled acetone droplets in a Leidenfrost state on liquid substrates, Phys. Fluids 29, 032103 (2017). 